
allemande

51-1 | 2019

La République démocratique allemande dans l'espace public européen (1949-2018)

\title{
Prekäre DDR-Repräsentation: Die Europa-Tourneen des Leipziger Gewandhausorchesters in den 1950er bis 1980er Jahren
}

Friedemann Pestel

\section{OpenEdition}

Édition électronique

URL : https://journals.openedition.org/allemagne/1384

DOI : 10.4000/allemagne.1384

ISSN : 2605-7913

Éditeur

Société d'études allemandes

Édition imprimée

Date de publication : 2 juillet 2019

Pagination : 83-97

ISSN : 0035-0974

Référence électronique

Friedemann Pestel, „Prekäre DDR-Repräsentation: Die Europa-Tourneen des Leipziger Gewandhausorchesters in den 1950er bis 1980er Jahren", Revue d'Allemagne et des pays de langue allemande [Online], 51-1 | 2019, Online erschienen am: 02 Juli 2020, abgerufen am 03 Juni 2022. URL: http://journals.openedition.org/allemagne/1384 ; DOI: https://doi.org/10.4000/allemagne.1384 


\title{
Prekäre DDR-Repräsentation: Die Europa-Tourneen des Leipziger Gewandhausorchesters in den 1950er bis 1980er Jahren
}

\author{
- Friedemann Pestel*
}

\section{Einleitung: Orchestertourneen zwischen DDR-Kulturbeziehungen und Risikodiplomatie}

Im Januar 1961 reiste das Leipziger Gewandhausorchester erstmals in die Volksrepublik Polen. Bei ihrer Besprechung des Gastspiels legte die Arbeiter- und Parteizeitung Trybuna ludu den Akzent ganz auf die tradierten Qualitäten des Orchesters: „Der Name ,Das Gewandhausorchester ist fast ein Synonym für die herrlichsten Traditionen der deutschen oder überhaupt der europäischen Musikkultur.“ Das Orchester bezwinge die Zuhörer „vor allem durch die einzigartige Kultur des Zusammenspiels, eine Kultur, die man nur in jahrzehntelanger Arbeit unter der Leitung hervorragender Künstler erreichen kann“(1). An dieser Charakterisierung sind mehrere Aspekte auffällig: Erstens wurde der Auftritt eines DDR-Orchesters in einem sozialistischen Land ohne jegliche sozialistischen Interpretamente kommentiert. Stattdessen verwies der prominente Traditionsbegriff auf die bürgerliche Musikkultur des 19. Jahrhunderts. Zweitens spielte auf polnischer Seite ebenso wenig der Nationalsozialismus als Deutungsmuster oder der Antifaschismus-Mythos als Gegenpol eine Rolle, obwohl die Geschichte des Gewandhausorchesters zwischen 1933 und 1945 solche Anknüpfungspunkte geboten hätte ${ }^{(2)}$. Drittens wurde die Aufführung mithilfe der Kategorie „Europa' in einen dezidiert transnationalen Interpretationsrahmen eingeschrieben, der über den Systemgegensatz des Kalten Krieges hinauswies: ,Europa' blieb hier keineswegs auf den Ostblock beschränkt und stand zugleich komplementär zur Kategorie

* Dr. Friedemann Pestel ist wissenschaflicher Mitarbeiter am Historischen Seminar der Albert-LudwigsUniversität Freiburg. Sein Dank gilt Anna Karla und Ann-Judith Rabenschlag für ihre Kommentare.

1 Trybuna ludu, 18.01.1961.

2 Zur Orchestergeschichte grundlegend Claudius Вӧнм, Neue Chronik des Gewandhausorchesters, 2 Bde., Altenburg, Kamprad, 2018/19. 
der Nation. Die Besprechung betonte gleichermaßen die ,deutsche 'Verwurzelung des Orchesters wie die ,polnische 'Teilhabe an seiner Geschichte, etwa durch Auftritte des Geigers Henryk Wieniawski im 19. Jahrhundert. Viertens standen die Tournee und Berichterstattung darüber in einer Kontinuität reisender musikalischer Ensembles seit dem späten 19. Jahrhundert. 1961 waren internationale Gastspiele Kernbestandteil des Musikbetriebs, auch in Polen, wo mittlerweile amerikanische und japanische Orchester gastiert hatten. Bezeichnenderweise war die Warschauer Nationalphilharmonie während des Gewandhaus-Gastspiels ihrerseits gerade auf einer USA-Tournee ${ }^{(3)}$.

Diese spezifische Mischung von Mobilität, Transnationalität und Traditionsverhaftung als Kernelement der kulturellen DDR-Auslandsrepräsentation bildet den thematischen Rahmen dieses Aufsatzes. Anhand der Europatourneen des Gewandhausorchesters als dem führenden Konzertorchester der DDR zwischen den 1950er und 1980er Jahren diskutiert er das Verhältnis der politischen Inszenierung der Gastspiele von offizieller DDR-Seite, dem „Eigensinn“ des Orchesters und den vielfältigen Aneignungen in den Zielländern. Indem die an sich immer ähnliche Praxis des Konzertierens im Ausland eine große Vielfalt an Aneignungsmustern und Bedeutungszuschreibungen hervorbrachte, lässt sich zeigen, dass genau in der Vielfalt der über musikalische Aufführungen verhandelten Interessen der beteiligten Akteure ein zentraler Antriebsfaktor für die hohe internationale Präsenz von DDR-Ensembles lag. Staats- und Parteiapparat, Konzertveranstalter und Musiker, vor allem aber Journalisten und Publika in den Zielländern luden die Tourneen sinnstiftend auf - häufig um den Preis der Eindeutigkeit ihres ,Erfolgs' und damit vor allem der Wirksamkeit kultureller Repräsentationsstrategien der DDR.

Mit diesem geweiteten Fokus knüpft der Aufsatz an jüngere Forschungen an, die das Verhältnis von Musik und Politik, von musikalischer Mobilität und Kulturdiplomatie dynamisch als Aushandlungsprozess zwischen staatlichen wie nichtstaatlichen Akteuren betrachten. Indem diese Arbeiten die Handlungsmacht der Beteiligten jenseits eines traditionellen Verständnisses von Kulturdiplomatie ,von oben' betonen, legen sie ein größeres Augenmerk auf die unintendierten Effekte der Aufführungen auf der Aufnahmeseite, die die performativen Erwartungen von staatlicher bzw. nationaler Repräsentation auf der Senderseite immer wieder unterliefen ${ }^{(4)}$. Wenn Didier Francfort in diesem Zusammenhang von „Risikodiplomatie“(5) spricht, so erstreckt sich das Problem von Kontrollierbarkeit und Kontrollverlust des DDR-Regimes über seine musikalischen Aushängeschilder nicht nur auf die unmittelbare und permanent virulente Gefahr der ,Republikflucht` von Musikern auf Tourneen, sondern vielmehr auf die Wirkungen kulturdiplomatischer Projekte insgesamt.

3 Leipziger Volkszeitung (LVZ), 19.01.1961; Głos Pracy, 19.01.1961.

4 Rebekah Ahrendt, Mark Ferraguto, Damien Mahiet (Hg.), Music and Diplomacy from the Early Modern Era to the Present, New York, Palgrave MacMillan, 2014; Jessica C.E. Gienow-Hecht (Hg.), Music and International History in the Twentieth Century, New York, Berghahn, 2015; Simo Miккоnen, Pekka Suutari (Hg.), Music, Art and Diplomacy. East-West Cultural Interactions and the Cold War, Farnham, Routledge, 2016.

5 Didier FrancForT, „Tournées musicales et diplomatie pendant la Guerre froide“, Relations internationales, 156 (2013), S. 73-86, hier S. 74. 
Eine solche Perspektive ist im Fall der DDR besonders relevant, da sie die Entsendung von Künstlern ins ,nichtsozialistische“ Ausland bis Anfang der 1970er Jahre als Praxis internationaler Anerkennungspolitik in Ermangelung einer souveränen Außenpolitik sowie anschließend zur Profilierung politisch-kultureller Eigenständigkeit betrieb ${ }^{(6)}$. Diese Funktionalisierung des internationalen Musikbetriebs seitens der DDR wird im Folgenden in Beziehung zu den Aneignungsmustern der Aufnahmeseite und den ihnen zugrundeliegenden politischen, aber auch ökonomischen, medialen und künstlerischen Eigenlogiken des Musikbetriebs gesetzt: Wen oder was reisende Orchester in der Wahrnehmung der Publika repräsentierten, entzog sich zu großen Teilen den Intentionen der Senderseite.

Darüber hinaus stützt sich die Untersuchung der Gewandhaus-Tourneen auf eine in den letzten beiden Jahrzehnten gewachsene und sich ausdifferenzierende Forschung zur kulturellen Identitätsbildung der DDR durch Musik einerseits sowie zu den internationalen (Kultur-)Beziehungen der DDR, vor allem mit westlichen und neutralen Ländern, andererseits ${ }^{(7)}$. Indem dieser Aufsatz bei den Mobilitätspraktiken der musikalischen Akteure ansetzt, stellt er die vorwiegend bilateral untersuchten Kulturbeziehungen in einen transnationalen Zusammenhang, der jeweilige Spezifika sichtbar macht, aber auch auf übergreifende Inszenierungs- wie Aneignungsmuster und künstlerische Routinen verweist.

Nach einem Überblick über die Tourneetopografien des Gewandhausorchesters zwischen westlichen und sozialistischen Ländern beleuchtet der Aufsatz verschiedene Formen der DDR-Inszenierung bei den Gastspielen und argumentiert, dass gerade

6 Jonathan L. YAEger, „The Leipzig Gewandhaus Orchestra and the Wages of Diplomatic Service“, in: Ahrendt/Ferraguto/Mahiet (Hg.), Music and Diplomacy (Anm. 5), S.69-81; Pierre Jardin, „Objectifs et outils de l'action culturelle à l'étranger de la République démocratique allemande (19491969)“, in: Antoine MARÈs (Hg.), Culture et politique étrangère des démocraties populaires, Paris, Institut d'études slaves, 2007, S. 13-44.

7 Siehe u.a. Laura Silverberg, „East German Music and the Problem of National Identity“, Nationalities Papers, 37 (2009), S.501-522; David G. Tompkins, Composing the Party Line. Music and Politics in Early Cold War Poland and East Germany, West Lafayette, IA, Purdue University Press, 2013; Elaine Kelly, Composing the Canon in the German Democratic Republic: Narratives of Nineteenth-Century Music, Oxford, Oxford University Press, 2014; Kyle Frackman, Larson Powell (Hg.), Classical Music in the German Democratic Republic. Production and Reception, Rochester, NY, Camden House, 2015; Gerd Dietrich, Kulturgeschichte der DDR, Göttingen, Vandenhoeck \& Ruprecht, 2018 sowie Charis Pöтніg, Italien und die DDR. Die politischen, ökonomischen und kulturellen Beziehungen von 1949 bis 1980, Frankfurt am Main, Peter Lang, 2000; Magda Martini, La cultura all'ombra del muro. Relazioni culturali tra Italia e DDR (1949-1989), Bologna, Il Mulino, 2007; Henning Hoff, Großbritannien und die DDR 1955-1973. Diplomatie auf Umwegen, München, Oldenbourg, 2003; Stefan BERGER, Norman LAPORTE, Friendly Enemies: Britain and the GDR, 1949-1990, New York, Berghahn, 2010; Ulrich PfEIL, Die anderen deutsch-französischen Beziehungen. Die DDR und Frankreich 1949-1990, Köln, Böhlau, 2004; Christian Wenkel, Auf der Suche nach einem „anderen Deutschland“. Das Verhältnis Frankreichs zur DDR im Spannungsfeld von Perzeption und Diplomatie, München, Der Gruyter Oldenbourg, 2014; Rolf PfeIffer, Die DDR und Österreich 1949-1972. Beziehungen in den Jahren offizieller Beziehungslosigkeit, Aachen, Shaker, 2015; Maximilian Graf, Österreich und die DDR 1949-1990. Politik und Wirtschaft im Schatten der deutschen Teilung, Wien, Verlag der österreichischen Akademie der Wissenschaft, 2016. Anknüpfungspunkte für eine transnationale DDR-Geschichte bietet Detlev BRUNNER, „DDR ,transnational‘. Die internationale Solidarität der DDR“, in: Alexander GAllus, Axel SCHILDT, Detlef Siegfried (Hg.), Deutsche Zeitgeschichte - transnational, Göttingen, Wallstein, 2015, S. 64-80. 
mit Blick auf das weitgehend kanonisierte Tourneerepertoire die Aufführungen ihre politische Spezifik viel häufiger verloren ${ }^{(8)}$. In dem Maße, in dem DDR-Politiker im Sinne einer inoffiziellen Anerkennungspolitik mit den Gastspielen auf den aktiven Einbezug der ausländischen Publika zielten und den Aufführungen soziale Mobilisierungswirkungen zuschrieben, lässt sich die Reichweite dieser Mobilisierungseffekte diskutieren. Abschließend betrachtet der Aufsatz dann im Sinne der „Risikodiplomatie“ die Auslandskonzerte eines DDR-Ensembles als Praktiken einer „deutschen und europäischen Musikkultur“ im variablen Verhältnis von Erwartung und Erfahrung in verschiedenen europäischen Ländern.

\section{Wachstumskurs durch Westtourneen}

Nach ersten Tourneen nach Westeuropa zwischen 1916 und 1931 entwickelte sich das Gewandhausorchester nach dem Zweiten Weltkrieg zu einem regelrechten Reiseorchester. Zwischen 1951 und 1989 absolvierte das Orchester fast 1000 Auslandskonzerte, die meisten davon in der Bundesrepublik, gefolgt von den USA und Japan ${ }^{(9)}$. Auf Europa entfielen zwei Drittel der Konzerte. Nur knapp 100 bzw. weniger als 10 Prozent der weltweiten Konzerte führten das Orchester in sozialistische Länder (d.h. Ostblock und Jugoslawien). Auf Europa bezogen bedeutete dies immer noch knapp sechsmal mehr Konzerte im Westen als im Osten. Diese Ungleichverteilung, die für die DDR-Gastspielpolitik insgesamt repräsentativ ist, erklärt sich in erster Linie aus der politischen Funktionalisierung der Tourneen für die DDR-Anerkennungspolitik sowie später zur kulturellen Abgrenzung gegenüber der Bundesrepublik ${ }^{(10)}$. Zudem wuchs ihre ökonomische Bedeutung zur Devisenbeschaffung ${ }^{(11)}$.

Diese Entwicklungen schlugen sich beim Gewandhausorchester quantitativ nieder. Gab es in der 1950er Jahren und infolge des Mauerbaus 1961 noch vereinzelt tourneelose Spielzeiten, wurden Auslandsgastspiele ab Mitte der 1960er Jahre zum integralen Bestandteil des jährlichen Orchesterbetriebs. Diese Routinen wurden nur noch punktuell, v.a. unmittelbar nach Chefdirigentenwechseln, unterbrochen. In den 1950er und 1960er Jahren spielte das Orchester im Saisondurchschnitt rund ein Dutzend Auslandskonzerte; in den 1970er Jahren erhöhte dieser sich auf 30, in den 1980er Jahren sogar auf über 40 Konzerte. Den absoluten Gipfelpunkt markierte die Spielzeit 1979/80 mit 65 Auslandskonzerten. Diese wachsende internationale Präsenz, zusätzlich zu den Leipziger Konzerten, den Operndiensten und den Aufführungen in der Thomaskirche, bedingte maßgeblich eine massive personelle Aufstockung des Orchesters von 100 Musikern nach dem Zweiten Weltkrieg auf 200 Planstellen in den 1980er Jahren ${ }^{(12)}$. Seine Reisetätigkeit machte das Gewandhausorchester zum zahlenmäßig größten Orchester der Welt.

8 Siehe auch Frank Trommler, Kulturmacht ohne Kompass. Deutsche auswärtige Kulturbeziehungen im 20. Jahrhundert, Köln, Böhlau, 2014, S. 686.

9 Gezählt werden die eigenständigen Konzerte des Gewandhausorchesters. Alle Angaben zu Daten und Programmen stammen aus der Konzertdatenbank des Gewandhausarchivs Leipzig.

10 Hans Lindemann, Kurt Müller, Auswärtige Kulturpolitik der DDR. Die kulturelle Abgrenzung der DDR von der Bundesrepublik Deutschland, Bonn, Verlag Neue Gesellschaft, 1974, S. 27.

11 J. YAEgER, „The Leipzig Gewandhaus Orchestra“ (Anm. 6).

12 Hans-Rainer Jung, Claudius Böнм, Das Gewandhaus-Orchester. Seine Mitglieder und seine Geschichte seit 1743, Leipzig, Faber \& Faber, 2006, S. 237. 


\section{Performanz der Mehrdeutigkeit: Anerkennungspraktiken und Repertoirepolitik}

In den 1950er und 1960er Jahren gastierte das Gewandhausorchester überwiegend in Ländern, zu denen die DDR keine diplomatischen Beziehungen unterhielt, folglich als Staat nicht anerkannt war und vor Ort nur eingeschränkt unterstützt wurde. Diese politische Konstellation stellte Orchester, Dirigent und die sich um 1960 als staatliche Alleinvertreterin für Gastspielabschlüsse durchsetzende Vorläuferorganisation der späteren Künstleragentur der DDR vor erhebliche logistische Herausforderungen, etwa bei der Visabeschaffung über das Allied Travel Office in Westberlin ${ }^{(13)}$. Zugleich nutzten die Organisatoren die sich trotz des Fehlens institutionalisierter bilateraler Kulturdiplomatie ergebenden politischen Spielräume. Bei der GroßbritannienTournee 1958, der ersten Ländertournee ins ,nichtsozialistische‘ Ausland jenseits der Bundesrepublik und der neutralen Schweiz, setzte die Gewandhausleitung bei der britischen Konzertagentur van Wyck durch, dass zu Beginn jedes Konzertes die britische Nationalhymne gespielt wurde, die das Publikum in althergebrachter Weise im Stehen anhörte ${ }^{(14)}$. Noch bevor sich das Publikum setzen konnte, folgte daraufhin die DDRHymne als klangliches Emblem der Abgrenzung gegenüber der Bundesrepublik. Die Manipulation des Publikumsverhaltens machte die Aufführung der Hymne zugleich zu einer indirekten Anerkennungsgeste gegenüber dem politisch nicht anerkannten Staat. Da zudem die BBC das Londoner Auftaktkonzert übertrug, wurde trotz Ablehnung seitens der Bundesregierung die DDR-Hymne bei dieser Gelegenheit erstmals vom Ausland aus gesendet. Diese öffentliche Sicht- und Hörbarkeit der DDR erfuhr durch Rathausempfänge an Konzertorten eine zusätzliche Aufwertung ${ }^{(15)}$. Daraufhin gab in der Bundesrepublik das Auswärtige Amt die Direktive aus, diese öffentliche symbolische Präsenz mithilfe ihrer Auslandsvertretungen zu unterbinden und insbesondere bei den Außenministerien zu erwirken, dass die Erteilung von Einreisegenehmigungen an Unterlassungsbedingungen geknüpft werde. Entsprechend untersagte das Foreign Office dann 1965 das Spielen der DDR-Hymne ${ }^{(16)}$.

Ein genauerer Blick auf die mediale und gesellschaftliche Einbettung der Europatourneen zeigt, wie die DDR in westlichen Ländern durch auswärtige Kulturpolitik den bundesrepublikanischen Alleinvertretungsanspruch weiter performativ unterlief. Als sich Mitte der 1960er Jahre in Frankreich die Kulturbeziehungen zu Ostdeutschland langsam liberalisierten, entsprach dies den Zielen der 1958 gegründeten und dem Parti communiste français nahestehenden Échanges franco-allemands, die vehement für eine Anerkennung der DDR eintraten und hierfür lokale Initiativen

13 Zur Künstleragentur, siehe die Erinnerungen ihres letzten Direktors Hermann FALK, Zu Gast in der Welt - Die Welt zu Gast. Die Künstleragentur der DDR - Fakten und Anekdoten zum internationalen Kulturaustausch, Berlin, Nora Verlag, 2015.

$14 \mathrm{Zu}$ den beginnenden Musikbeziehungen mit Großbritannien, Berger/Laporte, Friendly Enemies (Anm. 7), S. 128.

15 Sonntag, 18.05.1958; Birmingham Post, 02.05.1958; Bericht Berliner Theater- und Konzert-Agentur, 12.05.1958, Bundesarchiv Berlin-Lichterfelde (BAr) DR 1/178; Vermerk Auswärtiges Amt, 27.03.1958, Politisches Archiv des Auswärtigen Amtes (PAAA), B95/527; siehe auch J. YAEger, „The Leipzig Gewandhaus Orchestra" (Anm. 6), S.72f.

16 Auswärtiges Amt an Botschaften London und Brüssel, 28.01.1965, PAAA B 95/1165; Bericht, 23.11.1965, Gewandhausarchiv Leipzig (GHA) RA/1965-5. 
starteten $^{(17)}$. Eine besondere Rolle kam dabei Leipzigs Partnerstadt Lyon $\mathrm{zu}^{(18)}$. Entsprechend traten die EFA hier 1966 als Mitveranstalterin des Gewandhauskonzerts auf und organisierten danach einen Empfang. DDR-Symbole durften zwar auf Weisung der Pariser Regierung an die Präfektur nicht gezeigt werden, doch trat der konservative Bürgermeister Louis Pradel in einer Presseerklärung für die Normalisierung der Beziehungen zur DDR ein und argumentierte dabei mit der gemeinsamen Widerstandstradition beider Länder, die an den Antifaschismus-Mythos der DDR direkt anschlussfähig war ${ }^{(19)}$. Im neutralen Schweden hissten die Konzertveranstalter in Göteborg 1967 anlässlich des Gastspiels erstmals die DDR-Flagge vor dem Konzerthaus. Im NATO-Mitgliedsland Norwegen dankte wenige Tage später die Festivalleitung in Bergen der DDR-Regierung auf offener Bühne für die Mitwirkung des Orchesters ${ }^{(20)}$.

Ab den späten 1960er Jahren unterliefen die Gastspiele die Hallstein-Doktrin nicht mehr nur durch die Präsenz staatlicher Symbole; vielmehr trat die DDR durch Unternehmens- und Tourismuswerbung in den Programmheften als „das Land für Musikliebhaber" in Europa unmittelbar in Erscheinung ${ }^{(21)}$. Auch wenn es der DDR-Seite noch häufiger nicht gelang, diese Präsenz durchzusetzen, so ist dennoch eine gewisse Wirkungsreichweite der DDR-Inszenierung allein schon dadurch zu konstatieren, dass längere Tourneen jeweils fünfstellige Besucherzahlen sowie über Rundfunk, Fernsehen und Presse noch einen größeren Adressatenkreis zumindest angebotsseitig erreichten.

Für die DDR-Seite funktionierte die Auslandspräsenz als self-fulfilling prophecy: Mitreisende Vertreter staatlicher Institutionen und des SED-Apparats sowie Journalisten interpretierten die Konzerterfolge und symbolischen Gesten der Aufnahmeseite kausal als Anerkennung der kulturellen und politischen Leistungen der DDR insgesamt. In dieser Logik applaudierte dann das Publikum „dem Staat, aus dem dieser bedeutende Klangkörper kam“(22), und ergriff für dessen politische Anerkennung Partei. 1969 ging das Ministerium für Kultur so weit, den Gastspielen eine Funktion „zur Schaffung eines Systems der kollektiven Sicherheit in Europa“ zuzuweisen:

17 Ausführlich zu den EFA, C. Wenkel, Auf der Suche (Anm. 7), S. 35-99.

18 Allerdings war der Freundschaftsvertrag 1964 aufgrund der Direktiven der Pariser Regierung nicht zwischen den Städten, sondern den Freundschaftskomitees geschlossen worden; Thomas HöPEL, „Die Kunst dem Volke“. Städtische Kulturpolitik in Leipzig und Lyon 1945-1989, Leipzig, Leipziger UniVerlag, 2011, S. 295-305.

19 Bulletin d'information du comité du Rhône des échanges franco-allemands, Nr. 8, GHA RA/19663; Anneliese Syrbe, „Klingende Reise 5“, Azet, o.D., GHA RA/1966-3; Bericht Rudolf Gehrke (Rat der Stadt Leipzig), 18.08.1967, BAr DR 1/18580; BRD-Generalkonsulat Lyon an Auswärtiges Amt, 10.11.1966, PAAA B 95(ZA)/109980.

20 LVZ, 01.07.1967. Zum Flaggenkonflikt in Schweden, Alexander Muschiк, „Hammer, Zirkel, Ährenkranz - Die DDR-Symbole als Gegenstand deutsch-deutscher Auseinandersetzungen in Schweden“, in: Jan Hecker-Stampenl (Hg.), Nordeuropa und die beiden deutschen Staaten 1949-1989. Aspekte einer Beziehungsgeschichte im Zeichen des Kalten Krieges, Leipzig/Berlin, Kirchhof \& Franke, 2007, S. 149-158.

21 Programmheft Carlisle, 30.11.1970, GHA RA/1970-4; Bericht Rudolf Gehrke, 18.05.1967, BAr DR 1/18580; Programmhefte Schweiz, 1966, GHA RA/1966-3. 
„Sie beweisen gleichzeitig die Weltoffenheit der DDR im Geiste des proletarischen Internationalismus, des Humanismus und echter Völkerfreundschaft“(23). Gestützt wurden solche Schlussfolgerungen durch wohlwollende Konzertbesprechungen, die gerade in der linken Auslandspresse ebenfalls einen politischen Deutungsrahmen verwendeten. So schrieb die kommunistische dänische Zeitung Land og Folk 1967:

„Die Bresche, welche die konsequente Friedenspolitik der DDR, die hier bei uns von vorurteilsfreien Kräften unterstützt wird, in die antidemokratische Mauer gesprengt hat, welche die NATO um den deutschen Arbeiter- und Bauernstaat zu errichten suchte, wird immer größer. Sie ist jetzt so groß, dass das ganze Gewandhausorchester aus Leipzig hindurchschlüpfen [...] konnte“(24).

An solchen zugespitzten Äußerungen werden zwei Charakteristika des sozialistischen Tourneebetriebs deutlich: einerseits seine politische Wirkungsüberschätzung im Sinne einer unmittelbaren ideologischen Beeinflussung des ausländischen Publikums, andererseits aber die inhärente Mehrdeutigkeit der kulturellen Praktiken. Nur ein kleiner Teil der westlichen Konzertbesucher dürfte seine Karten aus politischen Sympathien für die DDR gekauft haben. Attraktiver waren dagegen die gerade in Repertoire und Interpretationspraxis augenfälligen bürgerlichen und traditionsverhafteten Routinen des Konzertbetriebs. Doch solange die Zuhörer Beifall spendeten oder gar Ovationen darbrachten und damit der DDR-Inszenierung nicht offen widersprachen, gaben sie die politische Deutungshoheit über ihr Verhalten zugleich weitgehend $\mathrm{ab}$ und ließen sich für weitergehende politische Schlussfolgerungen instrumentalisieren.

Im Umkehrschluss folgte aus dieser Deutungsoffenheit, dass die Performanz des Gewandhausorchesters in großen Teilen keine DDR-spezifische war - ein Befund, der für den Westen wie für den Ostblock gilt. Die Traditionsverhaftung des eingangs zitierten Beispiels aus Polen verweist auf solche übergreifenden Muster sowohl in den Aufführungs- wie den Rezeptionspraktiken. Im konkreten Falle hatten die DDRStellen daran sogar unmittelbaren Anteil, da sie ihren polnischen Partnern Werbematerial inklusive westlichen Pressestimmen zu den Gastspielen zur Verfügung gestellt hatten $^{(25)}$.

Ein zentraler Stellenwert für diese Konvergenzen kommt dem aufgeführten Repertoire zu, das auch bei DDR-Ensembles in der übergroßen Mehrheit im klassischromantischen Kanon des langen 19. Jahrhunderts deutsch-österreichischer Prägung verwurzelt blieb, im Falle des Gewandhauses mit einer gewissen Prominenz der Werke Mendelssohns oder Schumanns als Vertretern der, Musikstadt` Leipzig. Die für den Konzertbetrieb innerhalb der DDR angestrebte Quote von zunächst 25\%, später 15\% Werken von DDR-Komponisten wurde im Ausland mit Blick auf Veranstalterwünsche, Presseecho, Publikumsnachfrage und damit auch Einnahmen- und

23 Konzeption des Ministeriums für Kultur für die Verhandlungen der Künstleragentur der DDR zur Entsendung von DDR-Ensembles ins nichtsozialistische Ausland 1970/71, 19.11.1969, BAr DY 30/J IV 2/3/1579.

24 Land og Folk, 24.05.1967; dasselbe Motiv auf DDR-Seite bereits in LVZ, 30.01.1965.

25 Besprechung Ministerium für Auswärtige Angelegenheiten und Ministerium für Kultur, 31.08.1960, PAAA M 1 A 1732. 
Devisensicherung immer wieder unterschritten ${ }^{(26)}$. So lag der Anteil an Werken von in der DDR lebenden Komponisten in Großbritannien 1958 bei 4\%, in Polen 1961 bei $12 \%$, in Westeuropa 1966 immerhin bei 15\% und in Skandinavien 1967 sogar bei 17\%. Stießen die dem ,sozialistischen Realismus' verpflichteten Stücke generell auf wenig Zuspruch und wurden im Westen häufig als ästhetisch konservativ beurteilt, kam es auch zu peinlichen Missgriffen. 1958 spielte das Orchester in Großbritannien als repräsentatives Werk der ,progressiven` DDR-Musikkultur Ottmar Gersters Festliche Toccata, die die Presse als „überholt und drittklassig“ abtat, bis letztlich auffiel, dass Gerster das Stück bereits während des Nationalsozialismus komponiert hatte und somit das „bedauerliche Niveau demonstrierte, auf das deutsche Musik 1942 herabgesunken war“ “(27). Wurde dieser Fauxpas in der Tourneeauswertung des Kulturministeriums auch bemängelt, so stand das Stück weiterhin „als zeitgenössischer Beitrag aus unserer Republik" auf den Programmen ${ }^{(28)}$.

Der dominierende Komponistenkanon von Johann Sebastian Bach bis Richard Strauss wurde in ein teleologisches Erbe-Humanismus-Narrativ mit dem Zielpunkt der DDR-Musikpflege eingebaut und zugleich als Weltoffenheit verstanden ${ }^{(29)}$. Denn die DDR rechnete wie andere Länder damit, dass das traditionelle Repertoire zum Kernbestand der heimischen Orchester an den Gastspielorten gehörte und auch von anderen Tourneeorchestern, insbesondere bundesrepublikanischen, bedient wurde. Entsprechend spottete die Friedrich-Ebert-Stiftung, dass es der DDR-Hymne allein schon deshalb bedurfte, damit das Publikum die Herkunft des Orchesters überhaupt zur Kenntnis nahm ${ }^{(30)}$. Selbst von DDR-Seite revolutionär-progressiv aufgeladene Komponisten wie Ludwig van Beethoven oder Richard Wagner waren im europäischen Musikbetrieb seit dem 19. Jahrhundert so verankert, dass ihr Erfolg bei Publikum und Presse in erster Linie auf ihrer überkommenen Beliebtheit statt auf den konkreten Interpreten beruhte. Nur gelegentlich wurde ihre propagandistischideologische Vereinnahmung zum Thema. So maß etwa der Hampshire Telegraph 1965 die im Programmheft abgedruckte DDR-Deutung von Beethovens Freiheitsbotschaft am Bau der Berliner Mauer und stellte damit die völkerverbindende Kraft der Musik zwischen Ost und West wie auch den „revolutionären“ Charakter des SED-Regimes infrage ${ }^{(31)}$. Die zum Markenkern des Orchesters gehörenden Werke des einstigen Gewandhauskapellmeisters Felix Mendelssohn Bartholdy wollten DDR-Presse und SED-Funktionäre zwar immer wieder als Distinktionsgeste gegenüber einem bundesrepublikanisch-imperialistischen Antisemitismus verstanden

26 Zur Quote, David G. Tompkins, „Orchestrating Identity. Concerts for the Masses and the Shaping of East German Society“, German History, 30 (2012), S. 412-428, hier S. 422-424.

27 News Chronicle, 17.04.1958; The Sunday Times, 20.04.1958. Zu Gersters NS-Karriere, Fred K. PRIEBERG, Handbuch deutsche Musiker. 1933-1945, CD-ROM, 2009, S. 2055-2061.

28 Hans-Georg Uszkoreit (MfK) an Hans Pischner (stellvertretender Kulturminister), Leeds, 19.04.1958 sowie die nachfolgenden handschriftlichen Notizen, BAr DR 1/178 und den Aktenvermerk, 19.05.1958, PAAA M2 B 3328; für das Zitat Gewandhausintendant Werner Felix in LVZ, 02.06.1970.

29 Siehe auch Kyle Frackman, Larson Powell, „Introduction: Music and Heritage in the German Democratic Republic“, in: DIEs. (Hg.), Classical Music (Anm. 7), S. 1-19.

30 Lindemann/Müller, Auswärtige Kulturpolitik (Anm. 10), S. 128.

31 Hampshire Telegraph, 18.11.1965; ähnlich Basler Volksblatt, 27.11.1980. 
wissen; in der Rezeption dominierte dagegen der depolitisierende Lokalbezug zu Leipzig $^{(32)}$.

Insgesamt bestätigte insbesondere der wiederkehrende Traditions-Topos die Teilhabe des Gewandhausorchesters am europäischen Musikbetrieb, weniger hingegen die der $\mathrm{DDR}^{(33)}$. In den westeuropäischen Öffentlichkeiten wurde das Orchester häufig zu einer enträumlichten Exklave. So verwiesen die Presseberichte auf den universellen Reputationsgrad, den es sich bereits vor 1945 erworben hatte und auch in der Nachkriegszeit perpetuierte ${ }^{(34)}$. Daraus erklärt sich auch, dass während des Polen-Gastspiels die Krakauer Presse die Gewandhaus- „Tradition“ in eine Reihe mit den Berliner und den Wiener Philharmonikern stellte, obwohl diese in Krakau zuletzt zu Zeiten des nationalsozialistischen Generalgouvernements musikalisches ,Deutschtum ' hatten herausstellen sollen ${ }^{(35)}$. Dagegen zählten 1961 in Krakau Mendelssohn, Strauss und Beethoven als Komponisten, „die auch die Augen Europas auf das Gewandhaus lenkten“, wohingegen die DDR-Presse den Warschauer Jubel nach der Fünften Sinfonie „des großen deutschen Komponisten Ludwig van Beethoven“ damit erklärte, dass „die berühmten vier Töne ihres Grundthemas in der Zeit der Okkupation des Landes durch die Hitlerfaschisten als ein Signal der unausbleiblichen Befreiung galten “(36). In für den Tourneebetrieb charakteristischer Weise schlossen sich solche in ihren räumlichen Bezügen unterschiedlichen Aufladungen nicht aus, sondern bestätigten sich als routinisierte Teilhabe am internationalen Musikleben, jenseits der konkreten politischen Regime.

\section{Soziale Reichweite als Selbstbestätigung? Auf der Suche nach einem ,fortschrittlichen' Publikum}

Der kulturelle Internationalismus der DDR war auf Auslandsgastspielen im sozialistischen Selbstverständnis zwischen Nationalrepräsentation und humanistischem Universalismus nicht nur ein künstlerischer, sondern auch ein sozialer. Die Vorstellung einer sozialistischen Musikkultur bezog sich auf eine Demokratisierung des Musikzugangs. So beherbergte die DDR mit dem Gewandhausorchester nicht nur das größte Orchester, sondern war zugleich das Land mit der höchsten Orchesterdichte weltweit. Im Gewandhaus war man stolz, dass 1956 drei Viertel der Abonnements über volkseigene Betriebe verteilt wurden, wenngleich sich die tatsächlichen Konzertgänger eher aus Angestellten denn aus Arbeitern rekrutierten ${ }^{(37)}$.

32 Lothar Kusche, „England, Gewandhaus, Konwitschny“, Musik und Gesellschaft, 10 (1960), S. 364-366.

33 Zur Musikstadt siehe auch T. Höpel, „Die Kunst dem Volke“(Anm. 18).

34 Schwetzinger Zeitung, 01.06.1956; Westdeutsche Rundschau, 18.02.1957; Algemeen Handelsblad, 05.02.1957.

35 Friedemann Pestel, „Ein Programm, was auch irgend etwas über die Situation Deutschlands aussagt'? Wagner auf internationalen Orchestertourneen (1930er bis 1960er Jahre)“, in: Katharina Wagner, Holger von Berg, Marie Luise Maintz (Hg.), Sündenfall der Künste? Richard Wagner, der Nationalsozialismus und die Folgen, Kassel, Bärenreiter, 2018, S. 154-173 und 207-213, hier S. 163-165.

36 Dziennik Polski, 19.01.1961; LVZ, 16.01.1961.

37 Heinz Alfred Brocknaus, Musikgeschichte der Deutschen Demokratischen Republik. 1945-1976, Berlin, Verlag Neue Musik, 1979; Fritz Hennenberg, Das Leipziger Gewandhausorchester, Leipzig, Bibliographisches Institut, 1984 (5. Aufl.), S. 88. 
Die daraus abgeleitete soziale Vorbildwirkung erstreckte sich auch auf die Auslandstourneen. Deren Zuhörerschaft sollte idealerweise eine von Arbeitern und Jugendlichen sein. Angesichts der häufig nur bestenfalls mittelbar zu beobachtenden politischen Effektivität der Tourneen zugunsten der DDR verband sich mit einer möglichst egalitären Zusammensetzung des Publikums die Erwartung einer sozialen Wirksamkeit der Konzerte. Entsprechend waren die politischen und journalistischen Begleiter der Tourneen eifrig, aber meist mit mäßigem Erfolg damit befasst, in den Konzertsälen ,Werktätige und linke jugendliche Sympathisanten ausfindig zu machen: „[M]an sah den Menschen an, daß sie direkt von Fabriken und Büros ins Konzert kamen; man zieht sich im Konzertsaal die Jacke aus und raucht", so eine zufriedene Beobachtung aus dem nordenglischen Industrierevier $1958^{(38)}$. Wesentliche Ursache für die insgesamt begrenzten Mobilisierungseffekte lagen, gerade im westlichen Ausland, einerseits in der Verhaftung des klassischen Konzerts in habituellen bürgerlichen Distinktionsmustern seit dem 19. Jahrhundert ${ }^{(39)}$, andererseits in der Prestigewirkung internationaler Ensembles, die in teils eigenen Abonnementreihen mit erhöhten Eintrittspreisen auftraten, wenn nicht die Mitwirkung von Arbeiterorganisationen, linken Parteien oder DDR-Freundesgesellschaften für eine Öffnung sorgte. Auch dass die privaten Konzertagenturen ebenso westliche Künstler vertraten und aus Geschäftsgründen darauf verzichteten, das Orchester gezielter als DDREnsemble zu vermarkten, konterkarierte die Bemühungen um Sichtbarkeit mithilfe von DDR-Symbolik und DDR-Komponisten permanent.

Vom konservativen Repertoire abgesehen, war die Performanz des Gewandhausorchesters auf der Bühne eine ebenso wenig spezifisch sozialistische. Äußerlich stand das anfangs ausschließlich, später überwiegend männliche, im Frack spielende und die Konventionen des Konzertbetriebs genau befolgende Orchester weiterhin in der Traditionslinie des 19. Jahrhunderts. Doch auch abseits des Podiums entsprachen die Gewandhausmitglieder den Anforderungen an ,sozialistische Persönlichkeiten ' nur bedingt: Immer wieder beklagten DDR-Funktionsträger den „kleinbürgerlichen“ Habitus der Musiker, die von mitgebrachten Konserven lebten und sich ihre knapp bemessenen Devisen-Tagegelder im Wortsinn vom Munde absparten, um davon Konsumgüter zu kaufen, statt durch „Weltläufigkeit“ im Sinne einer „wirklich repräsentativen persönlichen Lebensweise als DDR-Bürger im Ausland“ systemische Überlegenheit $\mathrm{zu}$ demonstrieren ${ }^{(40)}$.

Indem im westlichen Ausland Publika, Repertoire und Musikerhabitus überwiegend bürgerlich, teilweise sogar exklusiv erschienen, gerieten die Konzerte, wie Journalisten nicht müde wurden zu betonen, an den Rand des performativen Selbstwiderspruches, weil sie entgegen ihren von DDR-Seite formulierten ideologischen Mobilisierungsannahmen konventionelle Routinen stabilisierten statt unterliefen ${ }^{(41)}$. Diese

38 Neues Deutschland (ND), 15.05.1958; siehe auch National-Zeitung, 13.05.1958.

39 Dazu Sven Oliver Müller, Das Publikum macht die Musik. Musikleben in Berlin, London und Wien im 19. Jahrhundert, Göttingen, Vandenhoeck \& Ruprecht, 2014.

40 Reisebericht Gewandhausorchester/Thomanerchor Schweiz, Luxemburg, Frankreich, 13.05.1954, Stadtarchiv (StA) Leipzig StVuR 8510 sowie Gerhard Stauch (Leiter der Zollverwaltung der DDR) an ZK SED, Abteilung Sicherheitsfragen, 29.12.1970, BAr DL 203/3278. 
Wirkungsumkehrung konnte im Extrem auf die DDR als Ganzes zurückfallen: 1979 spielte das Orchester in Florenz viermal hintereinander ein Beethoven-Bruckner-Programm, dessen Zusammenstellung für die Nachfrage nach dem Orchester wie für die Musealisierung des Repertoires bezeichnend war. Entsprechend schlussfolgerte die konservative Zeitung La Nazione, dass das Gewandhausorchester „die Atmosphäre des alten Deutschlands“ heraufbeschwöre: Infolge der „kulturellen Erstarrung in Ländern mit autoritären Regimen“ seien gerade diese „zu den Bewahrern der Klassiker“ geworden $^{(42)}$. In solchen Fällen verlor also die in der Tourneewerbung ständig apostrophierte „Pflege des humanistischen Kulturerbes“ jegliche progressive Assoziation. Bereits kurz zuvor hatte die Süddeutsche Zeitung die sozialen oder pädagogischen Effekte der Gastspiele negiert, indem sie ironisch fragte, ob die konservativen Programme am Ende ein „Trojanisches Pferd“ darstellten, um den dekadenten Kapitalismus vollends zu unterminieren ${ }^{(43)}$.

Trotz der gemessen an den DDR-Ansprüchen dürftigen gesellschaftlichen Bilanz, wäre es zu eindimensional, den Konzerten jegliche Mobilisierungswirkungen abzusprechen. Dazu gab es einerseits in Kooperation mit politischen Unterstützern signifikante Ausnahmen, andererseits entfalteten sich jenseits der kulturdiplomatischen Aufladungen auch auf lokaler Ebene Dynamiken. Unabhängig von oder auch konträr zu den DDR-Funktionalisierungen artikulierten Akteure der Aufnahmeseite über die Gastspiele lokale Interessen und Erwartungen an den Kulturbetrieb. So kritisierte die Presse in Wales anlässlich des Gastspiels 1970 das niedrige Niveau der regionalen Kulturförderung. In Toulouse dagegen galt der seltene Auftritt eines Gastorchesters 1987 als positive Wirkung der Dezentralisierung zugunsten der Regionen ${ }^{(44)}$.

Einen besonderen Fall bildet das postfranquistische Spanien, wo die Reorganisation und Öffnung des kulturellen Lebens, etwa durch Festivalgründungen und Gastorchester mit „europäischer" Klangidentität wie das Gewandhaus ${ }^{(45)}$, als internationale Teilhabe und Impuls für eigene Qualitätssteigerungen im kulturellen Angebot begrüßt wurden. Daran schrieben sich DDR-Kulturpolitiker indirekt einen Anteil zu, hatte doch eine Kammerformation des Gewandhauses bereits in Francos Todesjahr 1975 in Spanien gastiert. In der bereitwilligen Kooperation des Konzertagenten auf den Kanarischen Inseln erblickte der Kulturverantwortliche des Rats des Bezirks Leipzig damals bereits eine „politische Einsicht in die menschlichen, moralischen und kulturellen Werte der Länder des Sozialismus und in den [sic!] Grenzen des FrancoRegimes“(46). Beim Folgegastspiel des Gewandhauses auf den Kanaren 1988 stilisierte die Presse das Orchester vor dem eigenen Hintergrund der transición zur Demokratie zu einem Vertreter der Perestroika: Mithilfe Beethovens, der als musikalischer Revolutionär zum Sturz des Ancien Régime und zur "ganzheitlichen Bildung“ des Menschen beigetragen habe, ließen sich die politischen Reformbewegungen im Osten wie

42 La Nazione, 09.04.1979.

43 Süddeutsche Zeitung, 24.05.1976; siehe auch Badische Neueste Nachrichten, Karlsruhe, 11.01.1980; Nürnberger Nachrichten, 16.01.1980.

44 Wrexham Gazette, 03.12.1970; La Dépêche, o.D., GHA RA/1987-4.

45 Ritmo, 06/1984; ebenso $A B C, 01.02 .1988$.

46 Jochen Geldner (Rat des Bezirks Leipzig), Bericht Bachorchester Spanien/Kanaren, 12.06.1975, Sächsisches Staatsarchiv Leipzig, 20237/8011. 
im Süden als zwei Facetten eines auf aufklärerischen Werten beruhenden Neuordnungsprozesses in einem zwischen zwei bereits „beschädigten“ Systemen stehenden Europas verstehen, in dessen zukünftigen Aufbruch sich auch Spanien integrieren wollte $^{(47)}$. Dieselbe Fünfte Sinfonie, die der DDR-Presse 1961 in Polen als Symbol der Befreiung vom Nationalsozialismus und damit Weg in den Staatssozialismus gegolten hatte, kündigte 1988 in Spanien das Ende dieser Nachkriegsordnung an. Somit konnten die seinen kulturellen Repräsentanten zugeschriebenen künstlerischen Erfolge das DDR-Regime letztlich delegitimieren.

\section{Das Konzert als politisches Massenereignis: Beethovens Neunte Sinfonie bei der Fête de l'Humanité 1970}

Angesichts der schwierigen kulturdiplomatischen Wirkungskontrolle und der eingeschränkten sozialen Dynamiken der Tourneen legten die DDR-Organisatoren auf die Zusammenarbeit mit Sympathisanten und Unterstützern besonderes Augenmerk und betonten in einer Rhetorik von Frieden und Völkerfreundschaft den gemeinsamen musikalischen Bildungsauftrag. Im kommunistisch regierten Le Havre stellte die Kulturdezernentin 1966 unmissverständlich klar, dass Musikkonsum keine bürgerliche Unterhaltungspraxis darstellte, sondern ein mit didaktischer Strenge zu handhabendes Instrument der Massenbildung:

„Die Kluft zwischen der Isolierung vieler Kulturschaffender und der Unkultur der breiten Masse ist zu tief. [...] ,Die Kultur', sagt Picasso, ,ist wie Chinesisch, man muss sie erlernen'. Es braucht Lehrer zum Unterrichten, aber auch die aktive Mitwirkung des Schülers“(48).

Politisch umso wichtiger für die kulturelle Sichtbarkeit der DDR waren daher auch Gastspiele in linksregierten italienischen Städten, die ebenfalls eine Agenda der kulturellen Öffnung verfolgten, oder bei den großen Festen kommunistischer Zeitungen wie der Unità oder Humanité, die Massenpublika außerhalb der Konzertsäle anzogen ${ }^{(49)}$.

Die größte dieser Veranstaltungen bildete die Aufführung von Beethovens Neunter Sinfonie 1970 bei der Fête de l'Humanité im Bois de Vincennes vor 85.000 Zuschauern - mehr als sonst während der gesamten Spielzeit in Leipzig. Dafür übernahmen der PCF und das DDR-Kulturministerium die Kosten für Transport und Unterbringung von insgesamt 250 Orchestermusikern, Sängern der Rundfunkchöre Leipzig und Berlin und Solisten. Nichtsdestotrotz überwog bei Gewandhausleitung und -mitgliedern angesichts von Zugeständnissen bei der künstlerischen Qualität eines solchen OpenAir-Projekt die Skepsis gegenüber der einmaligen Gelegenheit, „den einfachen und werktätigen Menschen die 9. Sinfonie erstmalig zu Gehör zu bringen“ “(50). Anlass für die Einladung war Beethovens 200. Geburtstag, der im Zeichen seiner Deutung als

47 La Provincia, 27.01.1988.

48 Programmheft Le Havre, 08.11.1966, GHA RA/1966-3.

49 Programmheft Pavia, 06./08.09.1975, GHA RA/1975-4; ND, 11.09.1975; Bericht IM „Rudi Fischer“, 17.09.1970, Behörde des Bundesbeauftragten für die Stasi-Unterlagen (BStU), MfS BV Lpz. AIM 1825/92, Teil 2, Bd. 1; Hermann FALK, Direktive über die Teilnahme am Unità-Pressefest 1975, PAAA M2 B 1439/77.

50 Staatssicherheitsbericht des IM „Rudi Fischer“, einem Orchestermitglied, 17.09.1970, BStU MfS BV Lpz. AIM 1825/92, Teil A, Bd. 1 sowie Werner Felix (Gewandhausintendant) an die Künstleragentur der DDR, 20.04.1970, StA Leipzig GH 445. 
musikalischer Sachwalter der Französischen Revolution und Vorläufer Karl Marx’ sowie eines menschheitsumspannenden Universalismus im Sinne von Schillers „Seid umschlungen, Millionen" eine ideale Programmatik bot ${ }^{(51)}$.

Zwar spielte nach 1968 im musikalischen Programm der Fête Rockmusik eine immer größere Rolle, doch war ,klassische“ Musik ebenso fest verankert, da sie Künstler und Intellektuelle als PCF-Sympathisanten anziehen sollte und sich keineswegs auf zeitgenössische Komponisten wie Luigi Nono oder Krzysztof Penderecki beschränkte ${ }^{(52)}$. Indem das Musikprogramm als bewusst antikommerziell präsentiert wurde und eine große stilistische Bandbreite bot, bildete es das künstlerische Komplement zur politischen grande famille communiste, die der PCF abzudecken für sich in Anspruch nahm. Hinzu trat die vocation universelle der Fête als internationaler Veranstaltung, die sich auf ganz andere Räume und politisch-soziale Gruppen erstreckte, als es den üblichen Gewandhaus-Tourneeorten und ihren Publika entsprach: Themen der Fête 1970 waren der Vietnamkrieg, die Diktaturen in Spanien, Portugal und Griechenland wie der 100. Geburtstag Lenins ${ }^{(53)}$. Neben einer SED-Delegation waren auch das Neue Deutschland, die Échanges franco-allemands sowie Delegierte aus 35 ,Bruderparteien vertreten, und am Konzerttag forderte der PCF vor dem anwesenden Massenpublikum die Anerkennung der DDR ${ }^{(54)}$. Damit erhielt die wetterbedingt um den langsamen Satz verkürzte Beethoven-Aufführung bei allen künstlerischen Vorbehalten der Musiker einen kohärenten weltanschaulichen Deutungsrahmen, wie er in anderen Konzertsituationen faktisch nicht herzustellen war. Das Festpublikum kam dem Ideal einer globalen sozialistischen Internationale nahe wie sonst nie. Indem die Zuhörerschaft das sozialistisch-revolutionäre Beethoven-Bild der DDR in ihr Verhalten übersetzte, verhielt sie sich aus SED-Sicht geradezu exemplarisch, auch wenn sie die gängigen Konzertkonventionen unterlief: „Deutlich war die unterschiedliche Zusammensetzung des Publikums zu bemerken. Neben Intellektuellen saßen Arbeiter. Viele Jugendliche ,Beatles' und Gammler verfolgten die Aufführung mit außerordentlicher Aufmerksamkeit und Ruhe." Andernorts normwidriger Beifall nach jedem Satz stand hier für das Initiationserlebnis, „daß tausende dieses Werk [...] zum erstenmal hörten“. Anschließend drängten wiederum Tausende an den Bühnenrand „und hoben die Hände zum Zeichen der Sympathie und des Dankes“, wobei sie möglicherweise auch einfach nur Konventionen westlicher Rockkonzerte auf das ihnen unbekannte Klassikgenre übertrugen. Doch immerhin reckten "Arbeiter“ dann die Faust und skandierten „DDR“. Dirigent Kurt Masur wünschte dem PCF zukünftige Wahlerfolge, „damit es möglich

51 Werner KreceK, „Hinter der Tradition“, LVZ, o.D., GHA RA/1970-3; Paul MicheL, „Beethoven in unserer Zeit“, Musik und Gesellschaft, 20 (1970), S.9-13; siehe auch David B. Dennis, Beethoven in German Politics, 1870-1989, New Haven, Yale University Press, 1996, S. 177-191.

52 Zum Musikprogramm der Fête, Eric Drotт, „Music, the Fête de l'Humanité, and Demographic Change in Post-War France“, in: Robert Adlington (Hg.), Red Strains. Music and Communism outside the Communist Bloc, Oxford, Oxford University Press, 2013, S. 229-242; Noëlle GÉrôme, Danielle Tartakovsky, La fête de l'Humanité. Culture communiste, culture populaire, Paris, Messidor, 1988, S. 118 und 307-311.

53 Gerhard LEO, „Beethoven, die DDR und das Volk von Paris“, Horizont, o.D., GHA RA/1970-3; Karl Zumpe, „Das Fest der ,Humanitéc. DDR-Künstler spielten Beethoven in Paris“, Musik und Gesellschaft, 20 (1970), S. 805f.

54 ND, 18.09.1970; Auswärtiges Amt an Gesamtdeutsches Institut, 08.10.1971, PAAA B 95 (ZA)/109980. 
wird, ständig solche Werke vor den französischen Arbeitern auch in großen Pariser Konzertsälen aufzuführen“(55). Die Humanité jubelte, dass die Ode an die Freude nunmehr ihre „brüderliche Bestimmung“ erreicht habe, und die ostdeutsche Presse sah die DDR auf dem Weg zur Anerkennung einen weiteren Schritt vorangekommen ${ }^{(56)}$.

\section{Fazit: ,Europäische Öffentlichkeit' als Erwartung und Erfahrung kultureller Auslandsrepräsentation}

Ein solcher Deckungsgrad von politischen Erwartungen auf Sender- wie Empfängerseite, von Publikums- und Presserezeption sowie teilweise auch der Musikerreaktionen wie 1970 bei der Fête de l'Humanité stellte bei DDR-Auslandsgastspielen eine - wenn auch signifikante - Ausnahme dar. Die Regel war, dass die beteiligten Akteure auf beiden Seiten mit der Organisation und Durchführung der Konzerte ganz unterschiedliche Interessen verbanden, die sich jedoch nur selten explizit widersprachen. Die sich seit den 1950er Jahren entwickelnden Routinen des DDR-Gastspielbetriebs beruhten keineswegs auf einem Deutungskonsens über dessen politisch-kulturelle Spezifik bis hin zur Anerkennung der DDR, sondern auf der grundsätzlichen Deutungsoffenheit der musikalischen Praktiken. Diese Polysemie bot ausdrücklich Raum - und zumindest in der offiziellen DDR-Wahrnehmung auch Bestätigung - für die kulturdiplomatischen Erwartungen des SED-Regimes. Damit überwog der wahrgenommene Nutzen der Tourneen ihre ökonomischen Kosten wie den Unterhalt eines Riesenorchesters und ihre politischen Risiken, etwa die permanente Gefahr der „Republikflucht“ von Musikern auf Tournee. Bezeichnenderweise kehrte trotz verschärfter Kontrolle vom Humanité-Gastspiel eine Leipziger Chorsängerin nicht in die DDR zurück ${ }^{(57)}$. Umgekehrt boten die Gastspiele für die Öffentlichkeiten der Aufnahmeseite, aber auch für die beteiligten Musiker Gelegenheit zur Artikulation eigener Standpunkte, Interessen und eigensinniger Verhaltensweisen, allerdings nur selten im Dialog.

In der Gesamtschau auf die Europatourneen des Gewandhausorchesters lassen sich weder die Kontinuität noch die Ausweitung der Auslandspräsenz der DDR auf die Wirkungsabsichten des Staats- und Parteiapparats reduzieren. Diese Wirkung lässt sich gerade mit Blick auf die Anerkennungsfrage auch nicht kausal ermessen. Nichtsdestoweniger gewannen die Gastspiele eine empirische Materialität in Form von allein im Falle des Gewandhauses Hunderten von Konzerten sowie einer insgesamt siebenstelligen Zahl von Konzertbesuchern, die zur kulturellen Auslandsrepräsentation der DDR beitrugen und Staat und Regime zur internationalen Sichtbarkeit verhalfen.

Dabei gilt es jedoch zu berücksichtigen, dass zum einen die musikalischen Praktiken an sich wenig DDR-spezifisch waren und sich ab den 1960 zudem auch global weiteten. Sie beruhten auf den tradierten Institutionen, Formen und Repertoires des Musikbetriebs, die sich die DDR aneignete, ohne sie strukturell grundsätzlich zu verändern.

55 Bericht SED/Abteilung Kultur, 29.09.1970, BAr DY 30/IV A 2/9.06/158; Karl Zumpe, Die Geschichte des Gewandhausorchesters und die Probleme bei der Entwicklung des Klangkörpers zu einem führenden sozialistischen Musikinstitut der Deutschen Demokratischen Republik, Diss., Leipzig, 1980, S. 183.

57 Gerd Rossow (SED-Kulturabteilung) an Eberhard Fensch (SED-Agitationsabteilung), 30.06.1970, BAr DY 30/IV A 2/9.06/158; Bericht SED/Abteilung Kultur, 29.09.1970, BAr DY 30/IV A 2/9.06/158. 
Zum anderen gingen die Wirkungen der Tourneen nicht in bilateralen Kulturbeziehungen auf, sondern konstituierten sich als transnationale Mobilitätspraktiken, die ebenso lokale Reaktionen hervorriefen wie übergreifende Deutungsmuster ausprägten. Breit anschlussfähige Kategorien wie ,Tradition' und ,Europa erlaubten es, auf der Sender-, vor allem aber auf der Aufnahmeseite kulturelle Räume im Systemgegensatz als konvergent oder divergent $\mathrm{zu}$ imaginieren. Indem beide Dimensionen oftmals zugleich verhandelt wurden, ermöglichten diese Prozesse - komplementär zur Mobilität von DDR-Ensembles - die Teilhabe ihrer Kooperationspartner und Publika am europäischen Musikbetrieb.

Hatte 1961, kurz vor dem Mauerbau, eine polnische Arbeiterzeitung das Gewandhausorchester synonym zur „europäischen Musikkultur“ gesetzt, so stand am Ende dieser DDR-Mobilitätsgeschichte ebenfalls der Horizont ,Europa': Unter Vorsitz des EG-Präsidenten Jacques Delors und auf Initiative einer französischen katholischen Wochenzeitung wählte 1990 eine internationale Jury Gewandhauskapellmeister Kurt Masur aufgrund seiner und des Orchesters Rolle bei der ,friedlichen Revolution` 1989 zum „Europäer des Jahres“(58).

\section{Zusammenfassung}

Ab den 1950er Jahren entwickelte sich das Leipziger Gewandhausorchester zum musikalischen Aushängeschild der DDR. Mithilfe von Orchestertourneen, großenteils ins westliche Ausland, betrieb das DDR-Regime eine Politik der kulturellen Anerkennung und grenzte sich performativ von der Bundesrepublik ab. Allerdings folgten Publika, Konzertagenturen und Medien im Ausland dieser Inszenierung nur bedingt. Anstatt die DDR affirmativ zu repräsentieren, erwiesen sich die Konzerte als mehrdeutig, da sie in ihren Formaten und Programmen überwiegend langfristigen Kontinuitäten des europäischen Musikbetriebs folgten und da Akteure der Aufnahmeseite eigene Interessen artikulierten. Die Tourneeerfolge bemaßen sich weniger an einer spezifischen DDRKulturdiplomatie als an ihrer internationalen Anschlussfähigkeit.

\section{Résumé}

À partir des années 1950, l'Orchestre du Gewandhaus de Leipzig devient le fleuron musical de la RDA. Par les tournées de l'orchestre, surtout en Europe de l'Ouest, le régime est-allemand cherche à accélérer sa reconnaissance internationale et à se distinguer de la politique culturelle de la RFA. Les publics, agences de concert et médias étrangers sont cependant loin de suivre une telle mise en scène. Au lieu de constituer une représentation affirmative de la $R D A$, les concerts se révèlent ambigus. D'une part, les conceptions et les programmes s'adaptent aux continuités du marché musical européen. D'autre part, les acteurs des pays d'accueil formulent leurs propres attentes. Par conséquent, le succès des tournées doit être lu moins à l'aune d'une diplomatie culturelle spécifique de la RDA qu'en lien avec leur capacité à s'aligner sur les pratiques du secteur musical sur le plan international. 